production of the reaction. Nevertheless, the conclusion which I arrived at is that in extreme abduction the pupil of the abducted eye dilates, but that the ease with which this dilatation can be obtained varies within wide limits from case to case. W. Bevan Lewis, in a paper on "Ocular symptoms occurring in general paralysis of the insane" (Trans. Ophth. Soc., Vol. III., p. 204), concludes from the observation of 60 cases of general paralysis of the insane that the loss of reflex dilatation of the pupil on sensory stimulation is the earliest and most constant ocular sign in this condition. I had this observation in mind when examining my series of cases. When having my own eyes tested for Tournay's reaction, I experienced a feeling of strain and discomfort when the eyes were strongly lateralized, and this discomfort was confined to the abducted eye. This is not, of course, akin to cutaneous stimulation ; but as, in the majority of cases, it is only on extreme abduction that the reaction can be obtained, and as this extreme abduction causes discomfort, I thought that this might have something to do with the production of the reaction.

With regard to my series of 40 cases of general paralysis of the insane, the reaction was present in 15 , absent in 18 , doubtful in 4 , and unilateral in 3 cases. Of these 40 cases, 20 had Argyll Robertson pupils, and of these 20 the reaction was absent in 14 cases, present in 4 cases, and doubtful in 1 case. In 5 cases out of 40 the pupils did not react to light or accommodation; in these 5 cases the reaction was absent in 4 and present in 1 case. Of the 3 unilateral cases, in 2 cases the right pupil was inactive to light, while the left pupil reacted sluggishly; the right pupil was also larger than the left. In these cases the reaction was only obtained in the left eye. The other case was similar, with the exception that it was the right eye which had the smaller and active pupil and which showed the reaction.

\title{
NOTE ON WERNICKE'S PUPILLARY REACTION
}

\author{
BY \\ J. D. Cummins \\ DUBLIN
}

THE restricted usefulness of this reaction, from a clinical point of view, will, I think, be admitted. The theory of the reaction is proof against criticism. But the manner of performing the test is important.

If the region, from which the afterent impulse of the reflex arises, be confined to a relatively small area around the fovea, as Hess 
contends, the performance of the test requires a specially devised apparatus.

In the clinical round, one usually obtains a contraction of the pupil, by illuminating either half of the fundus with a light held at the side. This procedure is faulty.

More satisfactory is the plan of placing the patient, sideways, at a window, and, while directing him to look into the distance, of using diffuse daylight for illumination. Then, by turning right about, the other half of the fundus can be stimulated under like conditions. The eye, which is not being tested, ought to be covered with a dressing and light bandage.

For the test to be valid in the last procedure, the refraction of the eye must be emmetropic. Otherwise, the source of light and the illumined point on the retina will not be conjugate foci, and a diffuse lighting up, by reflection, of the interior of the globe will vitiate the result.

\section{ANNOTATIONS}

The value of letters and characters as visual tests.

That the letters used for sight testing vary in difficulty is a fact that has become almost universally recognized. Whereas letters like $\mathrm{L}$ and $\mathrm{J}$ which, approximate to isolated contours, are relatively easy, those which do not, such as $\mathrm{S}, \mathrm{R}$ and $\mathrm{B}$, are relatively difficult (cf. Hartridge and Owen, Brit. Jl. of Ophthal., Dec., 1922).

Dr. Ewing, before the International Congress at Washington (D.C.), quotes the visual values for both block and Gothic letters, as compared with the Landolt broken ring. These values were obtained by the ophthalmic section of the American Medical Association. The recommendations made by the latter are :-

(1) That block letters are superior to Gothic.

(2) That the following block letters of medium difficulty should be used:-T, V, U, C, O, Y, F, P, D, Z. N, E. R, S, C. H.

(3) That Snellen's original standard of 1 minute of arc for the width of the black lines composing a letter, and 5 minutes for the height and width be adhered to.

It is a matter for considerable regret that no information is given by Dr. Ewing as to the methods used in carrying out the tests. Were the tests done in ordinary day-light, i.e., under illumination of very variable intensity or was a carefully standardized light source of constant intensity employed? Were the patients referred to in the paper skilled at making visual comparisons, or were they hospital patients taken at random? Were the tests done with an 